\title{
A Multiaxial Layered Halide Double Perovskite Ferroelectric with Multiple Ferroic Orders
}

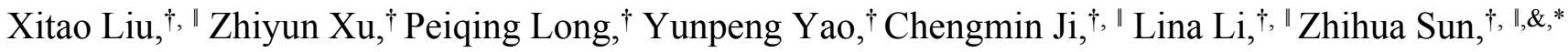
Maochun Hong ${ }^{\dagger}$ and Junhua Luo ${ }^{\dagger}, \|, \&, *$

${ }^{\dagger}$ State Key Laboratory of Structural Chemistry, Fujian Institute of Research on the Structure of Matter, Chinese Academy of Sciences, Fujian 350002, China

' Fujian Science \& Technology Innovation Laboratory for Optoelectronic Information of China, Fuzhou, Fujian, 350108, China

\&University of Chinese Academy of Sciences, Chinese Academy of Sciences, Beijing 100039, P. R. China 


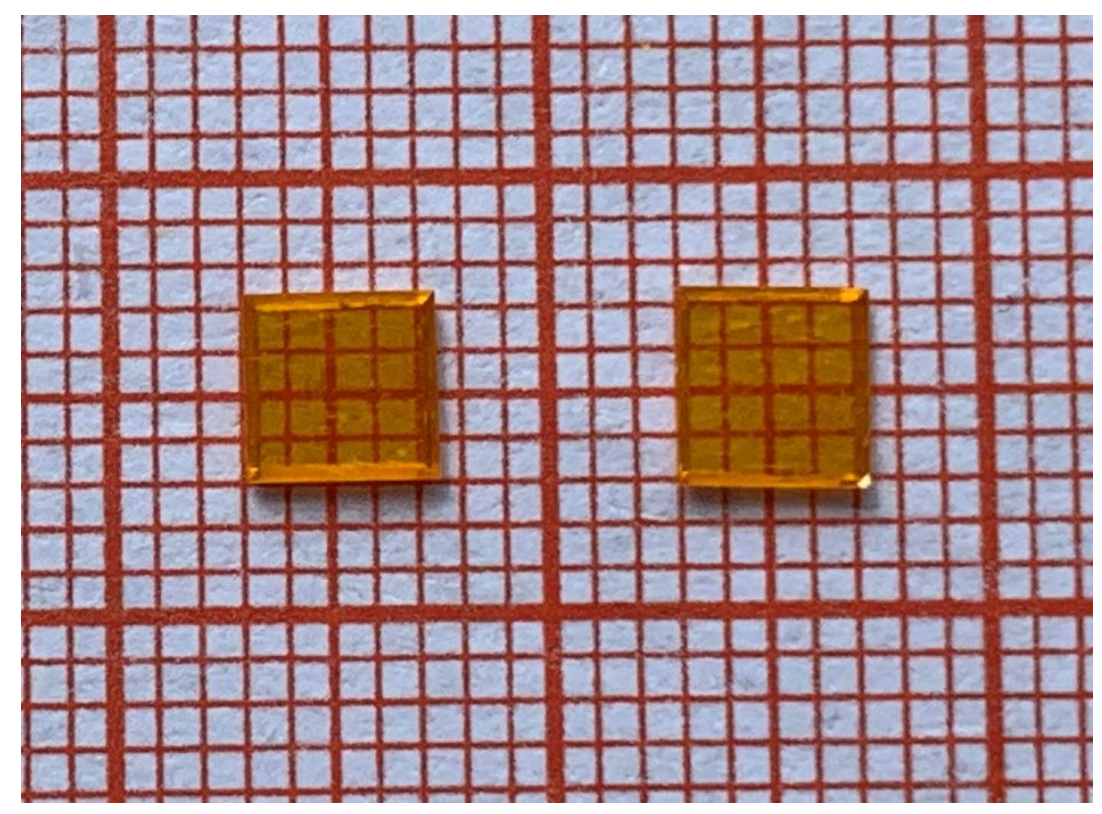

Figure S1. The grown single crystals of $(\mathrm{BA})_{2} \mathrm{CsAgBiBr}_{7}$. 


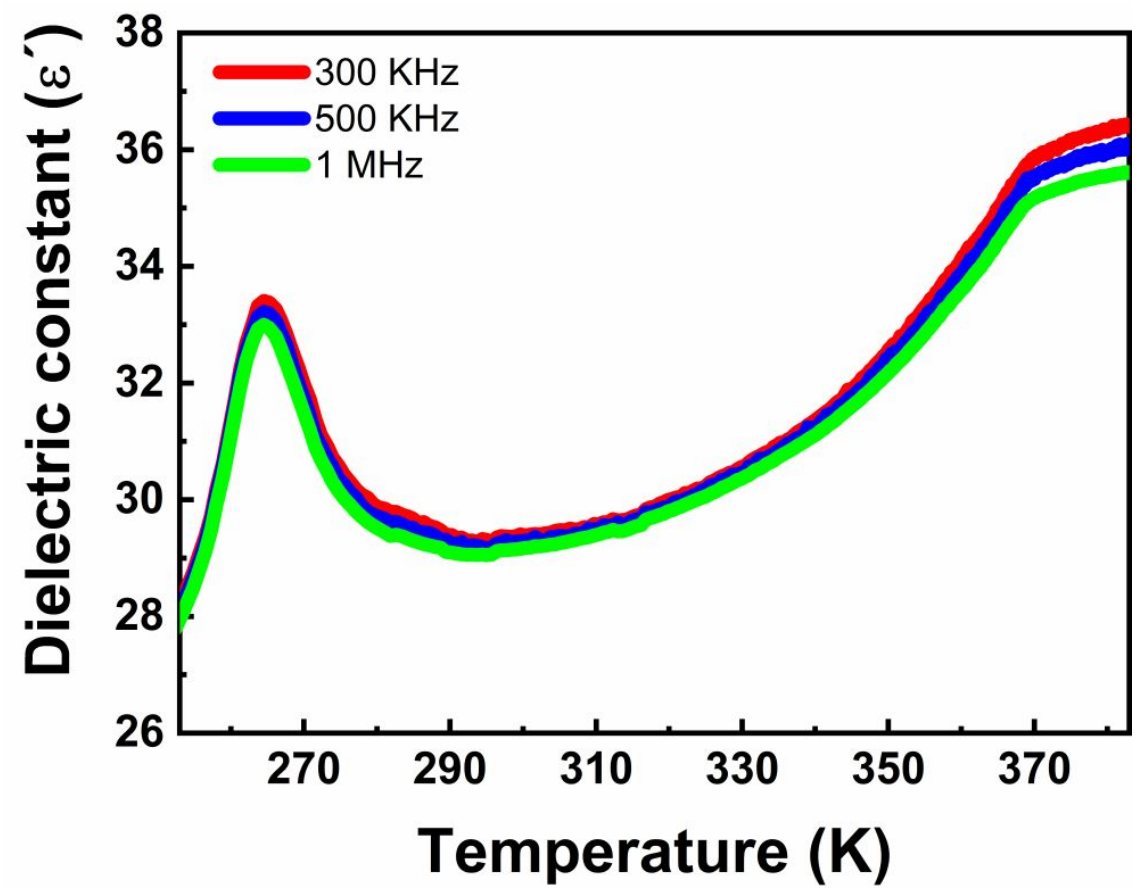

Figure S2. The temperature dependence of dielectric constants of powder sample of $(\mathrm{BA})_{2} \mathrm{CsAgBiBr}_{7}$. 


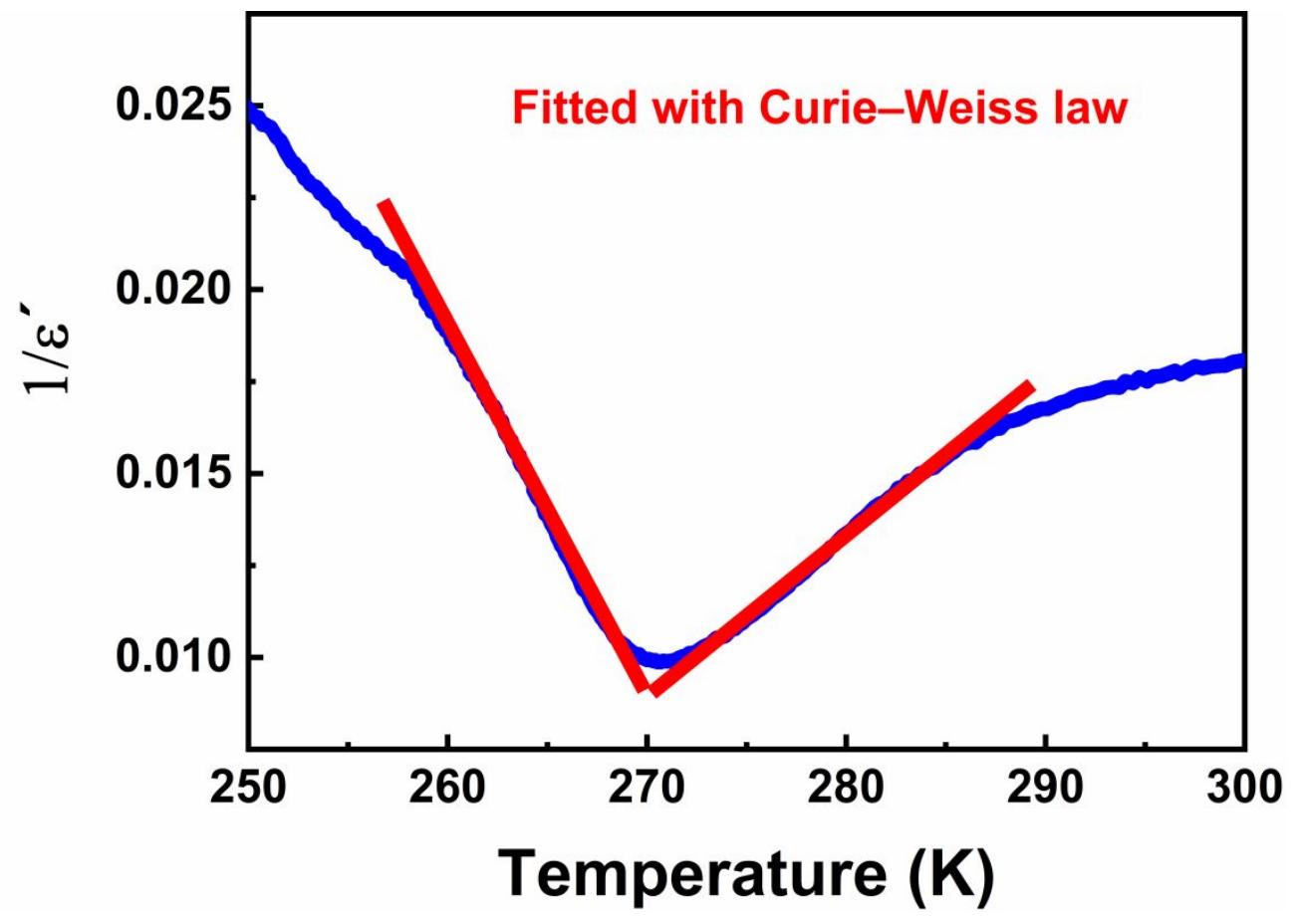

Figure S3. The fitting to Curie-Weiss law in the vicinity of $T_{\mathrm{c}}$ at $500 \mathrm{~Hz}$. 
(a)

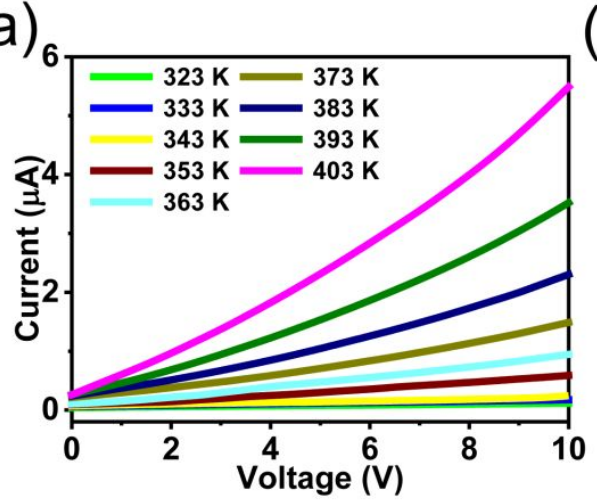

(b)

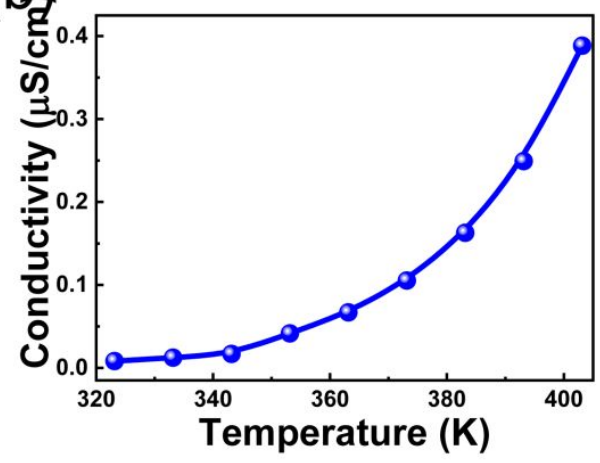

(C)

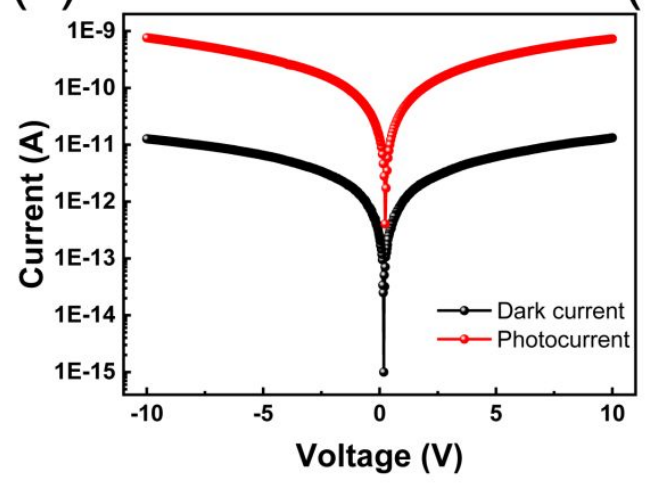

(d)

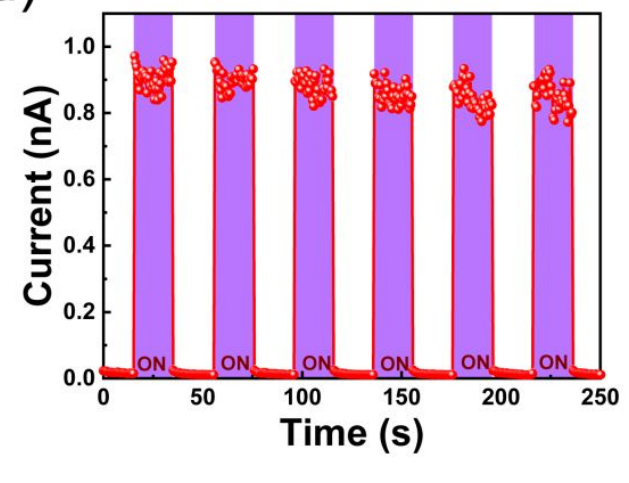

Figure S4. Photoelectric properties of 1: (a) $I-V$ curves at different temperature. (b) Variable-temperature conductivity. (c) $I-V$ curves in dark and under white light illumination $\left(100 \mathrm{~mW} / \mathrm{cm}^{2}\right)$. (c) Time-dependent reproducible photocurrent on/off switching under a $10 \mathrm{~V}$ bias. 


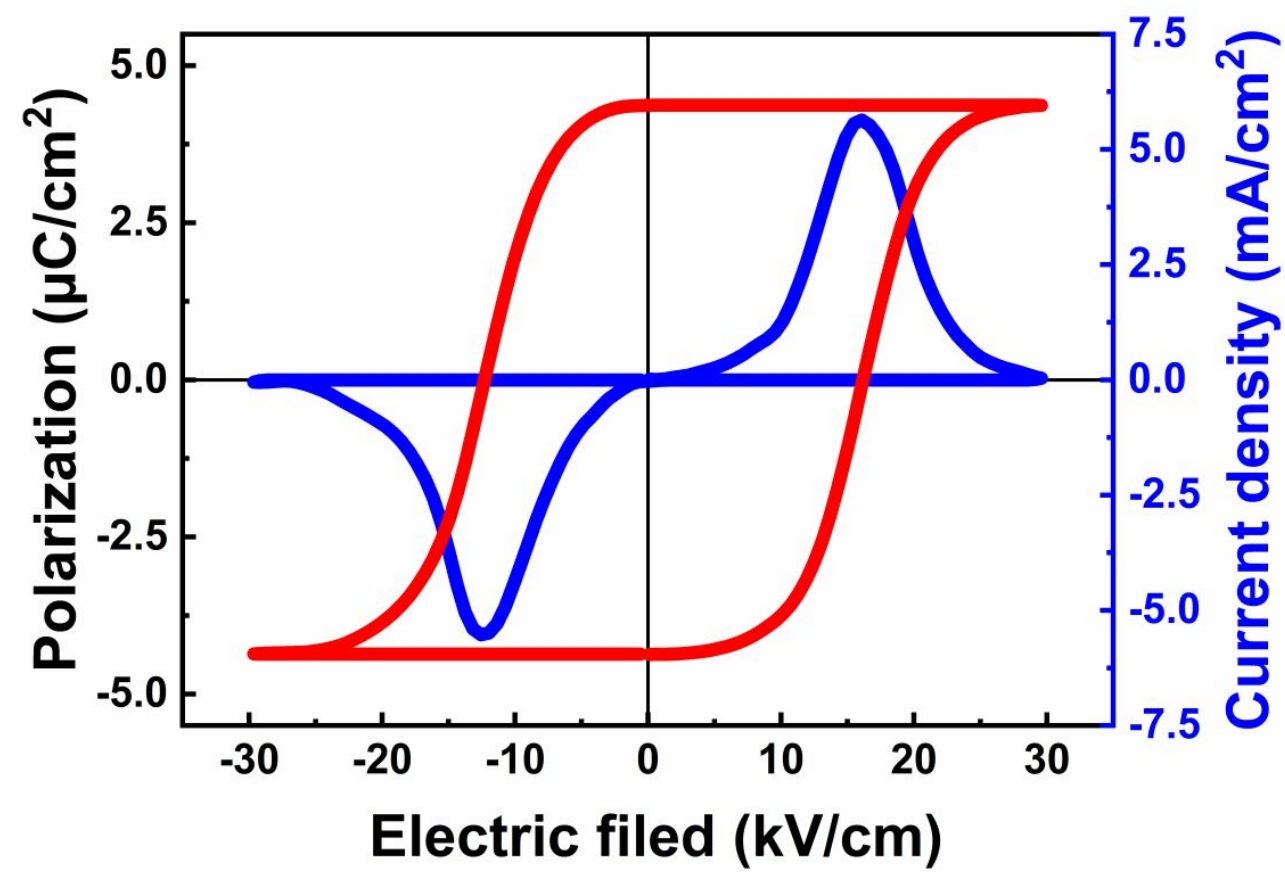

Figure S5: P-E hysteresis loops measured along the $\boldsymbol{b}$-axis via double-wave method at $253 \mathrm{~K}$. 


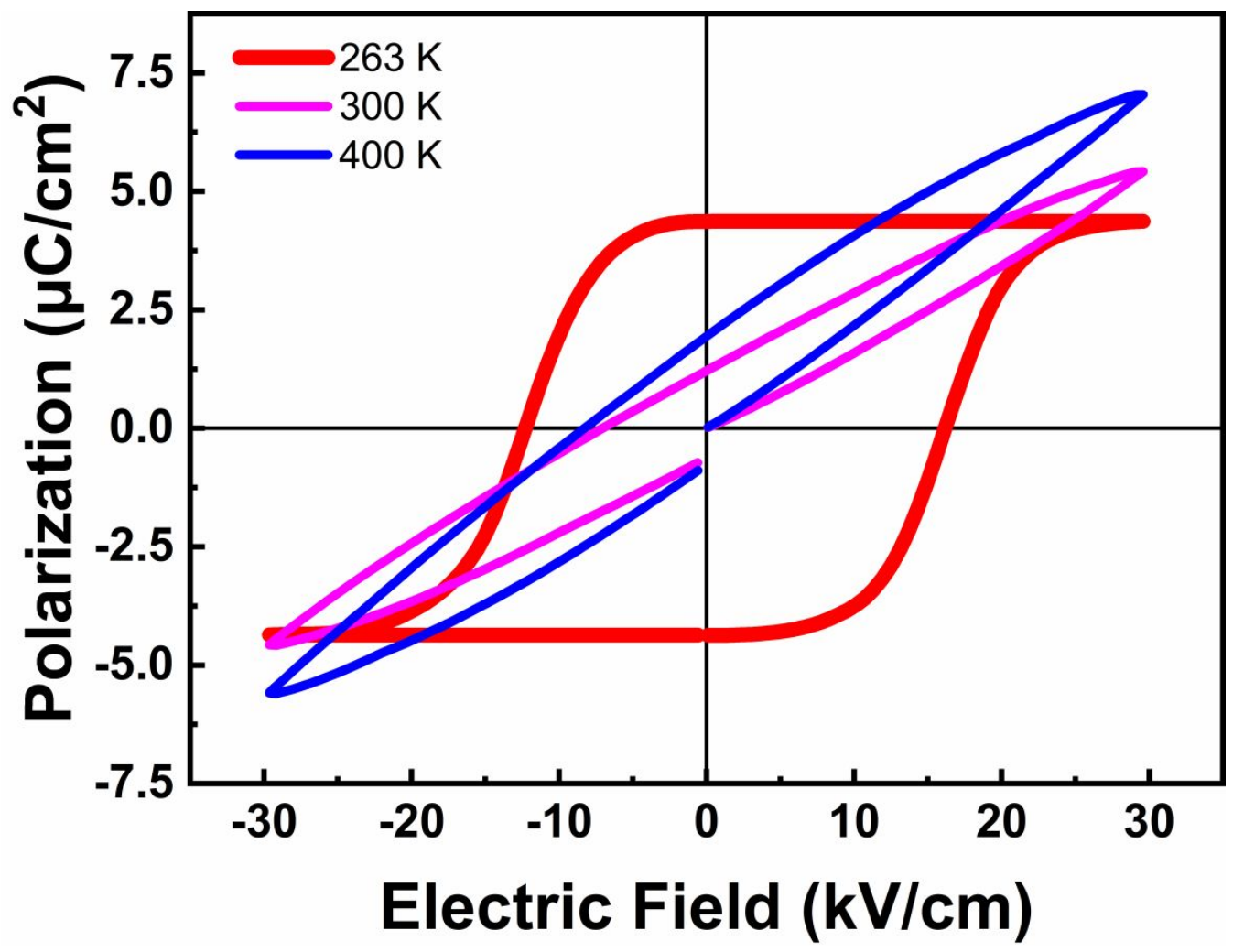

Figure S6: P-E hysteresis loops at LTP, ITP and HTP. 

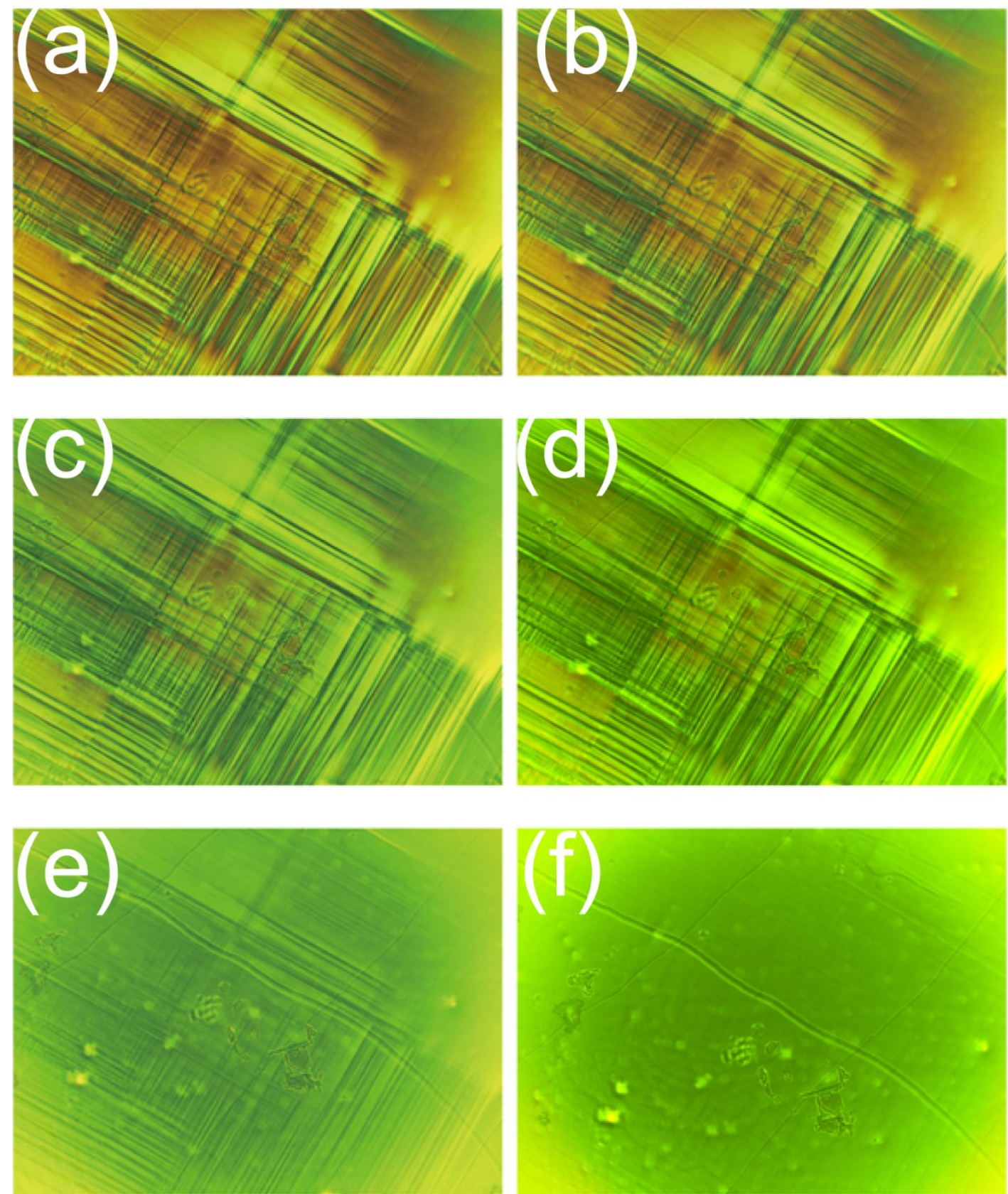

Figure S7: Temperature dependence of domain structures at P/A: 45 at different temperatures of (a) $250 \mathrm{~K}$, (b) $270 \mathrm{~K}$, (c) $320 \mathrm{~K}$, (d) $350 \mathrm{~K}$, (e) $370 \mathrm{~K}$, and (f) $400 \mathrm{~K}$ on heating (P/A reference direction [001]). 


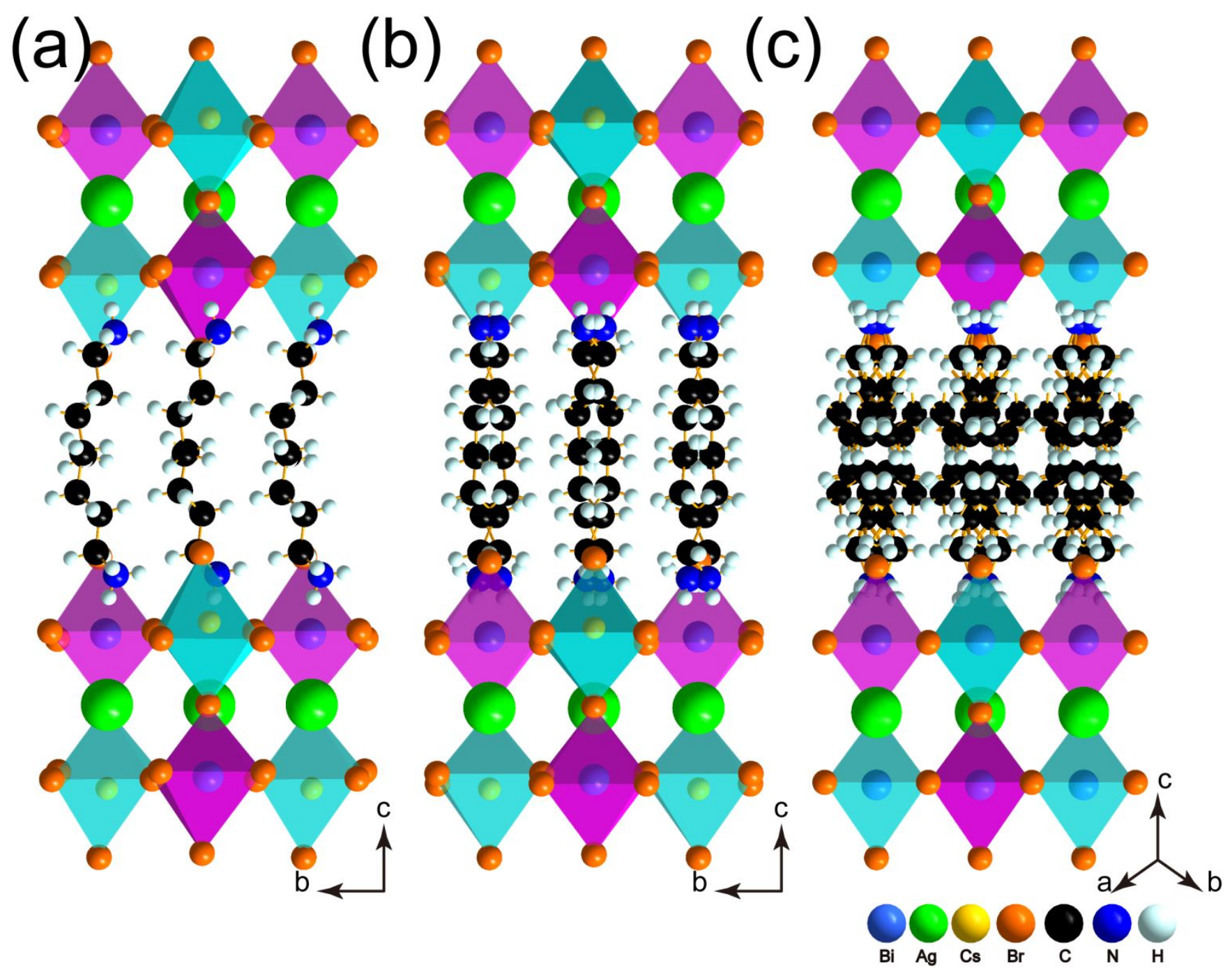

Figure S8. Crystal structures of 1 at different phases. (a) The 2D perovskite motif at LTP viewed along the $\boldsymbol{a}$-axis; (b) Packing diagram at ITP; (c) Packing structure at HTP. 
(a)

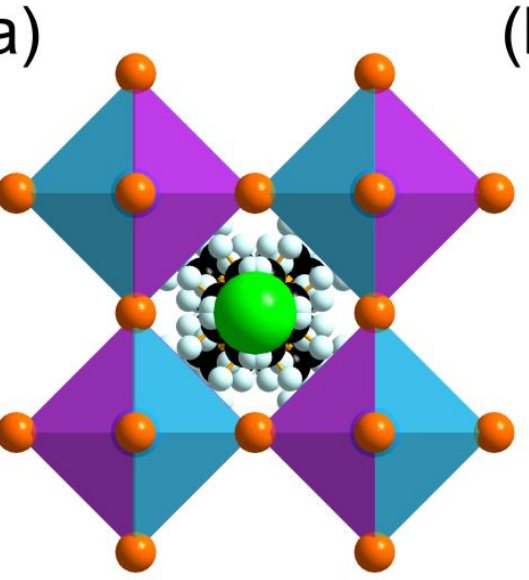

(b)

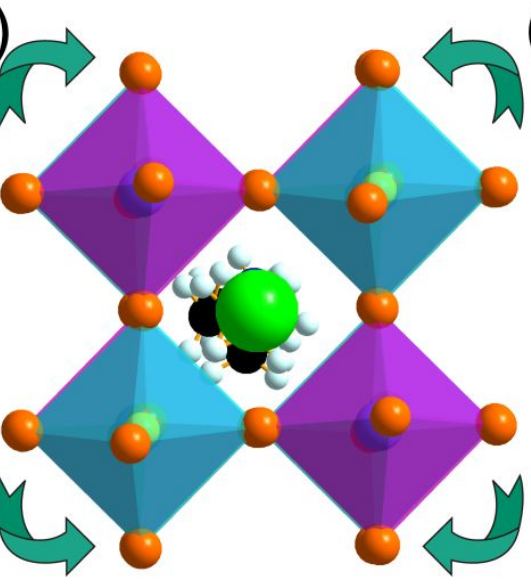

(c)

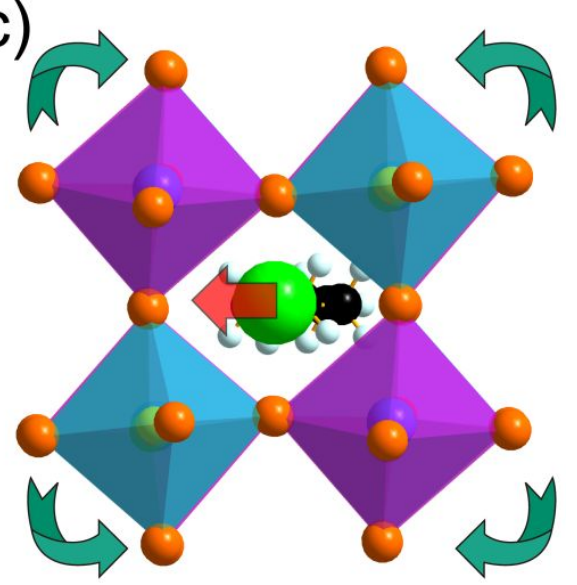

(d)

(e)
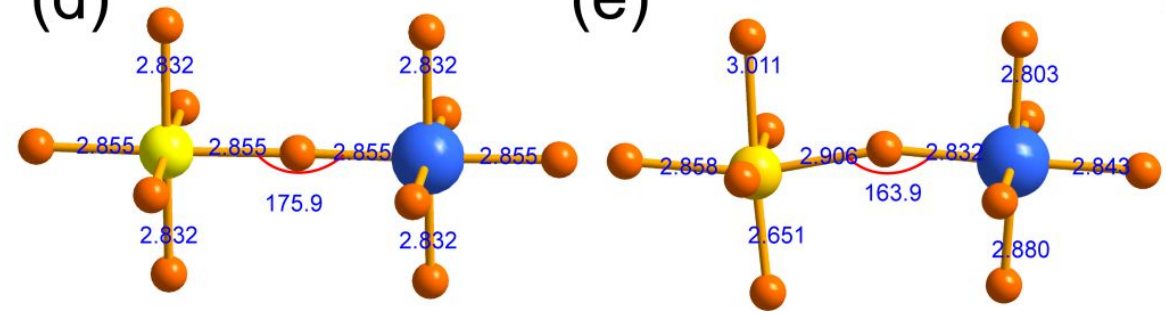

(f)

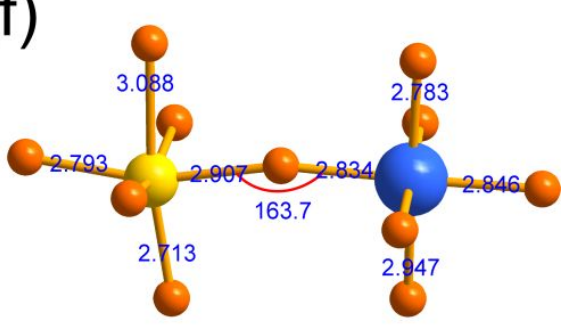

Figure S9. Two inhomogeneous octahedral configuration of $\mathrm{BiBr}_{6}$ and $\mathrm{AgBr}_{6}$ octahedra at $200 \mathrm{~K}(\mathrm{a}, \mathrm{d}), 298 \mathrm{~K}(\mathrm{~b}, \mathrm{e})$ and $400 \mathrm{~K}(\mathrm{c}, \mathrm{f})$. 


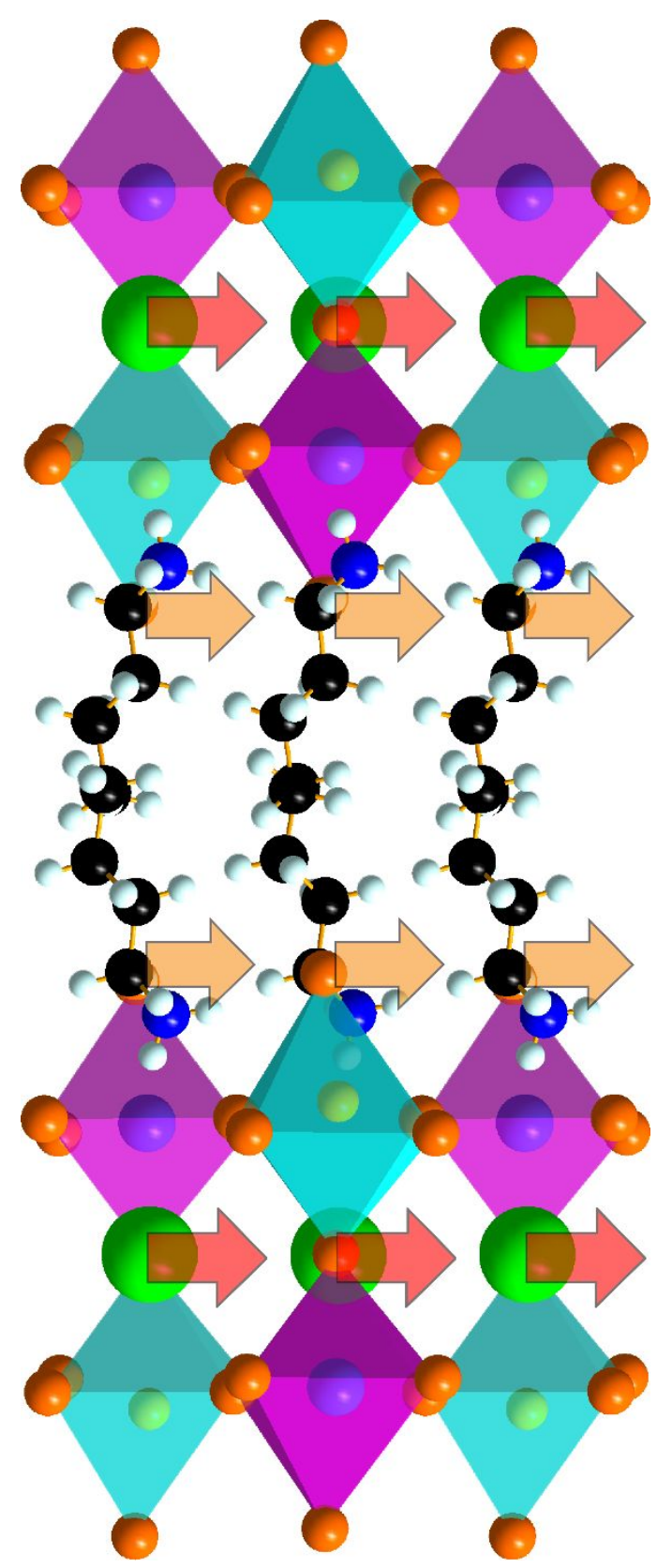

Figure S10. The 2D perovskite motif at LTP viewed along the $\boldsymbol{a}$-axis. The arrows denote a parallel array of dipoles related to dynamic orientation. 
ferroelectric phase

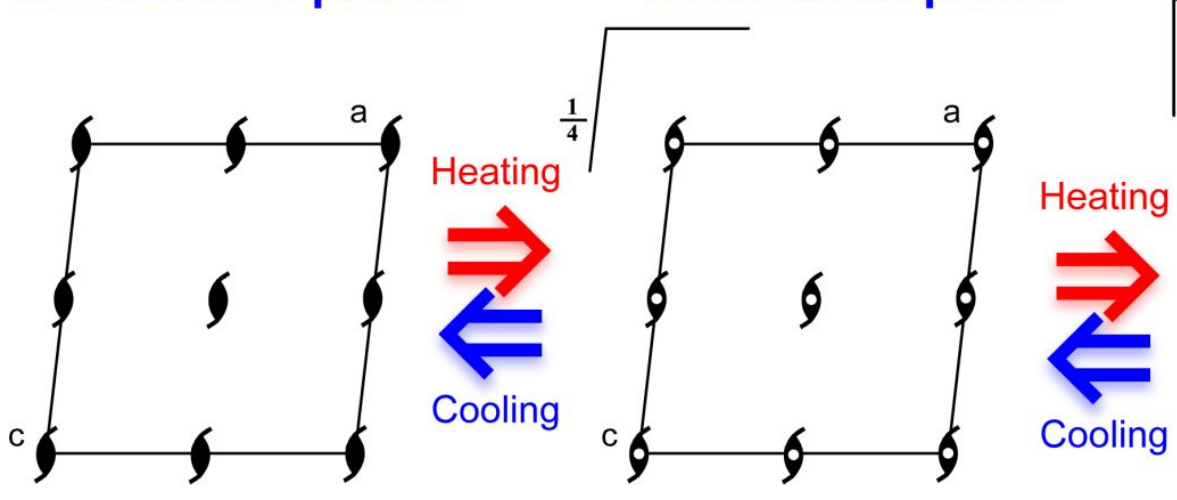

paraelectric phase

$\sqrt{\frac{1}{4}} \frac{1}{4}$

$111-\frac{111}{4}$

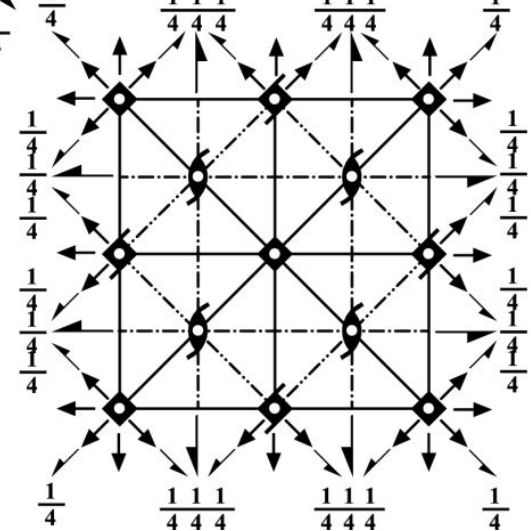

Figure S11: Symmetry breaking occurs of 1 from the ferroelectric phase (LTP) to ferroelastc phase (ITP) and then to the paraelectric phase (HTP) with a Shuvalov's symbol of $4 / \mathrm{mmmD} 2 \mathrm{~F} 2$. The symmetry operation elements from $16\left(E, 2 C_{4}, C_{2}, 2 C_{2}{ }^{\prime}, 2 C 2^{\prime \prime}, i, 2 S_{4}, \sigma_{h}, 2 \sigma_{v}, 2 \sigma_{d}\right)$ at the HTP to 4 $\left(E, C_{2}, \mathrm{I}, \sigma_{h}\right)$ at the ITP and finally to $2\left(E, C_{2}\right)$. 
Table S1 Crystal data for 1 collected at $200 \mathrm{~K}, 298 \mathrm{~K}$ and $400 \mathrm{~K}$.

\begin{tabular}{|c|c|c|c|}
\hline Empirical formula & $\mathrm{C}_{8} \mathrm{H}_{24} \mathrm{~N}_{2} \mathrm{BiAgCsBr}_{7}$ & $\mathrm{C}_{8} \mathrm{H}_{24} \mathrm{~N}_{2} \mathrm{BiAgCsBr}_{7}$ & $\mathrm{C}_{8} \mathrm{H}_{24} \mathrm{~N}_{2} \mathrm{BiAgCsBr}_{7}$ \\
\hline Formula weight & 1157.42 & 1157.42 & 1157.42 \\
\hline Temperature $(K)$ & 200 & 298 & 400 \\
\hline Space group & $P 2_{1}$ & $P 2_{1} / m$ & $I 4 / \mathrm{mmm}$ \\
\hline \multirow{6}{*}{ Cell parameters } & $\boldsymbol{a}=8.0618(5) \AA$ & $\boldsymbol{a}=8.0589(9) \AA$ & $\boldsymbol{a}=5.7059(5) \AA$ \\
\hline & $\boldsymbol{b}=7.8928(4) \AA$ & $\boldsymbol{b}=8.0388(9) \AA$ & $\boldsymbol{b}=5.7059(5) \AA$ \\
\hline & $\boldsymbol{c}=19.7697(12) \AA$ & $c=19.876(2) \AA$ & $c=40.293(6) \AA$ \\
\hline & $\alpha=90^{\circ}$ & $\alpha=90^{\circ}$ & $\alpha=90^{\circ}$ \\
\hline & $\boldsymbol{\beta}=101.734(2)^{\circ}$ & $\boldsymbol{\beta}=101.719(4)^{\circ}$ & $\boldsymbol{\beta}=90^{\circ}$ \\
\hline & $\gamma=90^{\circ}$ & $\gamma=90^{\circ}$ & $\gamma=90^{\circ}$ \\
\hline$V\left(\AA^{3}\right)$ & $1231.66(12)$ & $1260.8(2)$ & $1311.8(3)$ \\
\hline$Z, \rho_{\text {cal }} \cdot\left(\mathrm{g} / \mathrm{cm}^{3}\right)$ & $2,3.121$ & $2,3.049$ & $2,2.930$ \\
\hline$F(000)$ & 1032.0 & 1032.0 & 1032.0 \\
\hline Radiation $\lambda(\mathrm{Mo} \mathrm{K} \alpha)(\AA)$ & 0.71073 & 0.71073 & 0.71073 \\
\hline 2Theta range $\left(^{\circ}\right)$ & 5.162 to 49.974 & 5.068 to 49.998 & 7.212 to 54.994 \\
\hline \multirow{3}{*}{ Limiting indices } & $-9 \leq \mathrm{h} \leq 9$ & $-9 \leq \mathrm{h} \leq 9$ & $-7 \leq \mathrm{h} \leq 5$ \\
\hline & $-9 \leq \mathrm{k} \leq 9$ & $-9 \leq \mathrm{k} \leq 9$ & $-6 \leq \mathrm{k} \leq 7$ \\
\hline & $-23 \leq 1 \leq 23$ & $-23 \leq 1 \leq 23$ & $-50 \leq 1 \leq 52$ \\
\hline Reflections collected /unique & $18268 / 4363$ & $12704 / 2405$ & $3847 / 522$ \\
\hline Data/restraints/parameter & $4363 / 52 / 186$ & $2405 / 59 / 150$ & $522 / 61 / 62$ \\
\hline GOF & 1.060 & 1.066 & 1.094 \\
\hline \multirow{2}{*}{ Final $R$ indices $[I>2 \sigma(I)]^{\mathrm{a}}$} & $R_{1}=0.0358$ & $R_{l}=0.0560$ & $R_{l}=0.0545$ \\
\hline & $w R_{2}=0.0748$ & $w R_{2}=0.1347$ & $w R_{2}=0.1525$ \\
\hline \multirow{2}{*}{$R$ indices (all data) } & $R_{l}=0.0394$ & $R_{l}=0.0884$ & $R_{l}=0.0835$ \\
\hline & $w R_{2}=0.0764$ & $w R_{2}=0.1478$ & $w R_{2}=0.1703$ \\
\hline Largest diff. peak and hole, e $/ \AA^{-3}$ & $1.86,-1.29$ & $2.95,-2.23$ & $2.42 /-1.39$ \\
\hline Flack parameter & $0.282(10)$ & -- & -- \\
\hline
\end{tabular}

${ }^{\mathrm{a}} \mathrm{R}_{1}=\Sigma|| \mathrm{F}_{\mathrm{o}}|-| \mathrm{F}_{\mathrm{c}}|| \Sigma\left|\mathrm{F}_{\mathrm{o}}\right|, \mathrm{wR}_{2}=\left[\Sigma \mathrm{w}\left(\mathrm{F}_{\mathrm{o}}{ }^{2}-\mathrm{F}_{\mathrm{c}}{ }^{2}\right)^{2} / \Sigma\left(\mathrm{F}_{\mathrm{o}}{ }^{2}\right)^{2}\right]^{1 / 2}$ 
Table S1. Bond lengths for $(\mathrm{BA})_{2} \mathrm{CsAgBiBr}_{7}$ at $200 \mathrm{~K}$.

\begin{tabular}{|c|c|c|c|c|c|}
\hline Atom & Atom & Length/Å & Atom & Atom & Length/Å \\
\hline Ag1 & $\mathrm{Br}^{1}$ & $2.793(7)$ & Bi1 & Br5 & $2.783(3)$ \\
\hline $\operatorname{Ag} 1$ & $\mathrm{Br} 2^{2}$ & $2.831(7)$ & Bi1 & Br6 & $2.834(6)$ \\
\hline Ag1 & $\mathrm{Br} 3^{3}$ & $2.869(8)$ & $\mathrm{C} 1$ & N1 & $1.47(2)$ \\
\hline Ag1 & $\mathrm{Br} 5^{4}$ & $3.088(3)$ & $\mathrm{C} 1$ & $\mathrm{C} 2$ & $1.54(3)$ \\
\hline Ag1 & Br6 & $2.907(7)$ & $\mathrm{C} 2$ & C3 & $1.53(5)$ \\
\hline Ag1 & $\mathrm{Br} 7$ & $2.713(3)$ & C3 & $\mathrm{C} 4$ & $1.47(2)$ \\
\hline Bil & $\mathrm{Br} 1$ & $2.845(6)$ & $\mathrm{N} 2$ & $\mathrm{C} 5$ & $1.48(2)$ \\
\hline Bi1 & $\mathrm{Br} 2$ & $2.857(6)$ & $\mathrm{C} 5$ & C6 & $1.45(3)$ \\
\hline Bil & $\mathrm{Br} 3$ & $2.826(6)$ & C6 & $\mathrm{C} 7$ & $1.47(4)$ \\
\hline Bil & $\mathrm{Br} 4$ & $2.947(3)$ & $\mathrm{C} 7$ & $\mathrm{C} 8$ & $1.53(4)$ \\
\hline
\end{tabular}

Symmetry transformations used to generate equivalent atoms:

${ }^{1}-1+\mathrm{X}, 1+\mathrm{Y},+\mathrm{Z} ;{ }^{2}+\mathrm{X}, 1+\mathrm{Y},+\mathrm{Z} ;{ }^{3}{ }^{-} 1+\mathrm{X},+\mathrm{Y},+\mathrm{Z} ;{ }^{4}-\mathrm{X}, 1 / 2+\mathrm{Y},-\mathrm{Z}^{5}-\mathrm{X},-1 / 2+\mathrm{Y},-\mathrm{Z} ;{ }^{6} 1+\mathrm{X},+\mathrm{Y},+\mathrm{Z}$; 
Table S2. Bond angles for $(\mathrm{BA})_{2} \mathrm{CsAgBiBr}_{7}$ at $200 \mathrm{~K}$.

\begin{tabular}{|c|c|c|c|c|c|c|c|}
\hline Atom & Atom & Atom & Angle $/^{\circ}$ & Atom & Atom & Atom & Angle $/^{\circ}$ \\
\hline $\mathrm{Br}^{1}$ & Ag1 & $\mathrm{Br} 2^{2}$ & $91.1(2)$ & $\mathrm{Br} 5$ & Bil & $\mathrm{Br} 2$ & $88.37(17)$ \\
\hline $\mathrm{Br}^{1}$ & Ag1 & $\mathrm{Br} 3^{3}$ & $87.2(2)$ & $\mathrm{Br} 5$ & Bil & $\mathrm{Br} 3$ & $89.4(2)$ \\
\hline $\mathrm{Br}^{1}{ }^{1}$ & Ag1 & Br6 & $163.1(3)$ & $\mathrm{Br} 5$ & Bi1 & $\mathrm{Br} 4$ & $174.35(16)$ \\
\hline $\mathrm{Br} 2^{2}$ & Ag1 & $\mathrm{Br} 3^{3}$ & $162.5(3)$ & $\mathrm{Br} 5$ & Bi1 & Br6 & $88.80(18)$ \\
\hline $\mathrm{Br} 2^{2}$ & Ag1 & Br6 & $86.5(2)$ & Br6 & Bi1 & $\mathrm{Br} 1$ & $178.1(3)$ \\
\hline $\mathrm{Br} 3^{3}$ & Ag1 & Br6 & $90.1(2)$ & Br6 & Bi1 & $\mathrm{Br} 2$ & $88.7(2)$ \\
\hline $\mathrm{Br} 7$ & Ag1 & $\mathrm{Br} 1^{1}$ & $105.9(2)$ & Br6 & Bi1 & $\mathrm{Br} 4$ & $92.30(17)$ \\
\hline $\mathrm{Br} 7$ & Ag 1 & $\mathrm{Br} 2^{2}$ & $100.98(18)$ & $\mathrm{Ag} 1^{4}$ & $\mathrm{Br} 1$ & Bil & $172.0(3)$ \\
\hline $\mathrm{Br} 7$ & Ag1 & $\mathrm{Br} 3^{3}$ & $96.2(2)$ & $\mathrm{Ag} 1^{8}$ & $\mathrm{Br} 2$ & Bi1 & $162.2(3)$ \\
\hline $\mathrm{Br} 7$ & Ag 1 & Br6 & $90.94(19)$ & Bi1 & $\mathrm{Br} 3$ & $\mathrm{Ag} 1^{6}$ & $174.0(3)$ \\
\hline $\mathrm{Br} 1$ & Bil & $\mathrm{Br} 2$ & $91.51(16)$ & Bi1 & $\mathrm{Br} 5$ & $\mathrm{Ag} 1^{5}$ & $172.80(13)$ \\
\hline $\mathrm{Br} 1$ & Bil & $\mathrm{Br} 4$ & $89.60(18)$ & Bi1 & Br6 & Ag1 & $163.7(3)$ \\
\hline $\mathrm{Br} 2$ & Bil & $\mathrm{Br} 4$ & $86.12(15)$ & C6 & $\mathrm{C} 5$ & $\mathrm{~N} 2$ & $113(2)$ \\
\hline $\mathrm{Br} 3$ & Bil & Br1 & $89.6(2)$ & $\mathrm{C} 5$ & C6 & $\mathrm{C} 7$ & $112(3)$ \\
\hline $\mathrm{Br} 3$ & Bil & $\mathrm{Br} 4$ & $96.11(19)$ & N1 & $\mathrm{C} 1$ & $\mathrm{C} 2$ & $112(2)$ \\
\hline $\mathrm{Br} 3$ & Bil & Br6 & $90.16(16)$ & $\mathrm{C} 3$ & $\mathrm{C} 2$ & $\mathrm{C} 1$ & $110(3)$ \\
\hline $\operatorname{Br} 5$ & Bil & $\mathrm{Br} 1$ & 89.31(19) & $\mathrm{C} 4$ & $\mathrm{C} 3$ & $\mathrm{C} 2$ & $116(3)$ \\
\hline
\end{tabular}

Symmetry transformations used to generate equivalent atoms:

${ }^{1}-1+\mathrm{X}, 1+\mathrm{Y},+\mathrm{Z} ;{ }^{2}+\mathrm{X}, 1+\mathrm{Y},+\mathrm{Z} ;{ }^{3}-1+\mathrm{X},+\mathrm{Y},+\mathrm{Z} ;{ }^{4} 1+\mathrm{X},-1+\mathrm{Y},+\mathrm{Z} ;{ }^{5}-\mathrm{X},-1 / 2+\mathrm{Y},-\mathrm{Z} ;{ }^{6} 1+\mathrm{X},+\mathrm{Y},+\mathrm{Z} ;{ }^{7}-\mathrm{X}, 1 / 2+\mathrm{Y},-\mathrm{Z} ;{ }^{8}+\mathrm{X},-1+\mathrm{Y},+\mathrm{Z}$ 